\title{
A versatile magnetic refrigeration test device
}

\section{Bahl, Christian Robert Haffenden; Petersen, Thomas Frank; Pryds, Nini; Smith, Anders}

\section{Published in:}

Review of Scientific Instruments

Link to article, DOI:

$10.1063 / 1.2981692$

Publication date:

2008

Document Version

Publisher's PDF, also known as Version of record

Link back to DTU Orbit

Citation (APA):

Bahl, C. R. H., Petersen, T. F., Pryds, N., \& Smith, A. (2008). A versatile magnetic refrigeration test device. Review of Scientific Instruments, 79(9), 093906. https://doi.org/10.1063/1.2981692

\section{General rights}

Copyright and moral rights for the publications made accessible in the public portal are retained by the authors and/or other copyright owners and it is a condition of accessing publications that users recognise and abide by the legal requirements associated with these rights.

- Users may download and print one copy of any publication from the public portal for the purpose of private study or research.

- You may not further distribute the material or use it for any profit-making activity or commercial gain

- You may freely distribute the URL identifying the publication in the public portal

If you believe that this document breaches copyright please contact us providing details, and we will remove access to the work immediately and investigate your claim. 


\title{
A versatile magnetic refrigeration test device
}

\author{
C. R. H. Bahl, ${ }^{1, a)}$ T. F. Petersen, ${ }^{1,2}$ N. Pryds, ${ }^{1}$ and A. Smith ${ }^{1}$ \\ ${ }^{1}$ Fuel Cells and Solid State Chemistry Department, Ris $\phi$ National Laboratory for Sustainable Energy, \\ Technical University of Denmark, DK-4000 Roskilde, Denmark \\ ${ }^{2}$ DTU MEK, Building 402, Technical University of Denmark, Nils Koppels Allé, DK-2800 Lyngby, Denmark
}

(Received 12 June 2008; accepted 22 August 2008; published online 18 September 2008)

\begin{abstract}
A magnetic refrigeration test device has been built and tested. The device allows variation and control of many important experimental parameters, such as the type of heat transfer fluid, the movement of the heat transfer fluid, the timing of the refrigeration cycle, and the magnitude of the applied magnetic field. An advanced two-dimensional numerical model has previously been implemented in order to help in the optimization of the design of a refrigeration test device. Qualitative agreement between the results from model and the experimental results is demonstrated for each of the four different parameter variations mentioned above. (C) 2008 American Institute of Physics. [DOI: 10.1063/1.2981692]
\end{abstract}

\section{INTRODUCTION}

In recent years a number of devices that produce refrigeration by means of the magnetocaloric effect have been presented. Presentations of a number of the most recent of these may be found in Ref. 1 and a concise review of the devices may be found in Ref. 2. Magnetic refrigeration potentially offers advantages in the form of reduced energy consumption, low noise level, and nontoxic refrigerants as well as no ozone depletion potential. However, many of the magnetocaloric materials that have been proposed for use in magnetic refrigeration devices rely on expensive rare earth elements. ${ }^{3-5}$ This is also true, to an even greater extent, of the permanent magnet $(\mathrm{NdFeB})$ magnetic field source, where the price of Nd is the limiting factor. Recently, much work has gone into achieving the greatest cooling power when using a minimum amount of permanent magnet material while still maintaining a large field. ${ }^{6,7}$ The majority of the proposed magnetocaloric materials have modest magnetocaloric effects at room temperature in applied fields that may reasonably be achieved in a magnetic refrigeration device. Thus, care must be taken to ensure an optimal utilization of the temperature increase and decrease offered by the materials during magnetization and demagnetization.

The present paper describes parameter variation experiments carried out on a small but versatile magnetic refrigeration test device. This apparatus was designed to allow for an easy access and exchange of any parts as well as control and adjustability of any experimental parameters. This allows for a wide range of optimization work to be performed. However, the focus of this paper is not an optimization of the temperature span or coefficient of performance, rather it is to make reliable and robust contact between experiment and a numerical model based directly on the basic physics of the system.

An important tool in the optimization of any magnetic refrigeration device is a comprehensive and flexible numeri-

\footnotetext{
${ }^{a)}$ Electronic mail: christian.bahl@risoe.dk.
}

cal model. This may help in predicting the effect of a proposed alteration of the operation of the device as well as physical changes to the device. It also allows parameter optimizations to be performed consistently and efficiently.

In Secs. IV and V results from a previously published numerical two-dimensional (2D) model ${ }^{8,9}$ are compared to results obtained from an experimental magnetic refrigeration device. A good qualitative and, to some extent, quantitative agreement between the model predictions and experimental results will be demonstrated.

The device presented in the following is a linear design, relying on the active magnetic regenerator (AMR) cycle. Conventionally the AMR cycle consists of the following four steps: ${ }^{5,10,11}$ magnetization, resulting in an increase in the refrigerant temperature; removal of the heat from the refrigerant by the heat transfer liquid (cold-to-hot blow); demagnetization, resulting in a decrease in the refrigerant temperature; transfer of the heat from the heat transfer fluid to the refrigerant (hot-to-cold blow).

\section{THE MAGNETIC REFRIGERATION TEST DEVICE}

The experimental apparatus consists of a cylindrical plastic block, $40 \mathrm{~mm}$ in diameter. The block houses the AMR in a hole with a rectangular profile. This hole has slits either side to hold sheets of magnetocaloric material, as shown in Fig. 1(a). In the present experiments the AMR is loaded with 13 flat sheets of $0.9 \mathrm{~mm}$ thick gadolinium $(\mathrm{Gd})$, with a separation of $0.8 \mathrm{~mm}$ between each sheet. The Gd is $99.9 \%$ pure obtained from China Rare Metal Material Co. Each sheet is $25 \mathrm{~mm}$ wide and $40 \mathrm{~mm}$ along the flow direction, giving a total mass of $\mathrm{Gd}$ of $92 \mathrm{~g}$. Heat transfer fluid is moved along the AMR through the channels created by the separation of the $\mathrm{Gd}$ sheets. At either end of each Gd sheet, $20 \mathrm{~mm}$ long plastic flow guides ensure a laminar flow of the heat transfer fluid [see Fig. 1(b)]. Both the Gd sheets and the plastic flow guide sheets are flat and held in place by precision machined grooves in the plastic block, so that each channel has the same cross section. Perspex tubes, with an inside diameter of 


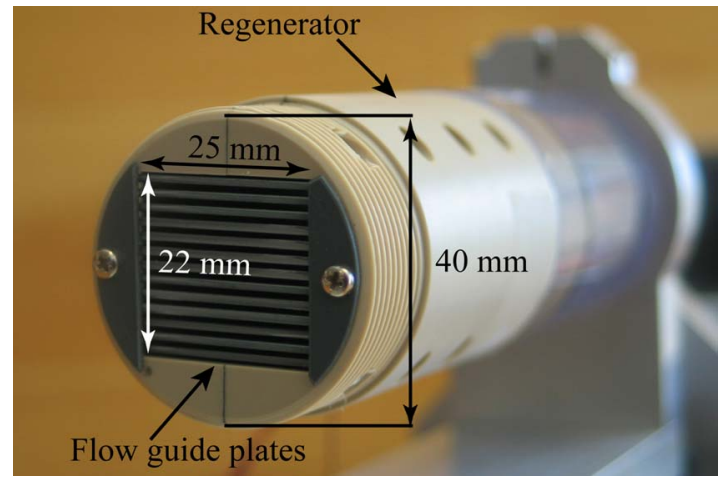

(a)

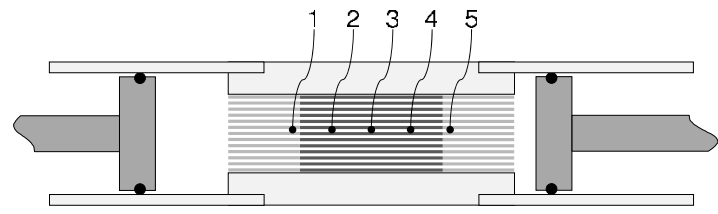

(b)

FIG. 1. (Color online) The experimental magnetic refrigeration apparatus. (a) Image of the AMR housed in a cylindrical plastic block. (b) Schematic cross section along the fluid flow direction. The Gd sheets are shown in dark gray while the plastic flow guides are shown in paler gray. The positions of the five thermocouples are indicated in the figure by the numbers $1-5$.

$34 \mathrm{~mm}$, are fitted to either end of the AMR block. Plastic pistons moving within these tubes force the heat transfer fluid through the AMR. The two pistons are rigidly fixed to a piece of aluminum such that they always move in phase. The distance and velocity of the piston movement are controlled by a stepper motor. The Perspex tube at the "cold end" of the AMR is insulated with insulating foam in order to minimize the heat losses to the environment through the tube outer surface. Due to geometrical constraints, the Perspex tube at the "hot end" was not insulated.

In order to measure the temperature gradient along the regenerator five type- $E$ thermocouples have been mounted equidistantly along the heat transfer fluid channel that is adjacent to the central Gd sheet. Each thermocouple protrudes $1 \mathrm{~mm}$ into the channel. The three central thermocouples are in the part of the AMR containing the Gd sheet; while the outer thermocouples on either side are in the region of the AMR with plastic sheets [see Fig. 1(b)]. Thermocouples protruding further into the channels, to avoid any effect of being close to the boundary with the plastic, were tested both experimentally and through numerical simulations. Due to the proximity to the Gd sheets at any point in the channel no significant boundary effect of a protrusion of $1 \mathrm{~mm}$ was observed, so this protrusion was chosen to avoid any blocking of the flow.

The whole AMR assembly is moved in and out of a magnetic field using a drive shaft controlled by a stepper motor. In the present experimental setup a LakeShore EM7 electromagnet capable of giving a maximum applied field of $\mu_{0} H=1.4 \mathrm{~T}$ is used. The sheets are oriented so the magnetic field is parallel to the plane of the sheets, thus minimizing the effect of demagnetization. A permanent magnet field source has recently been implemented, as will be described

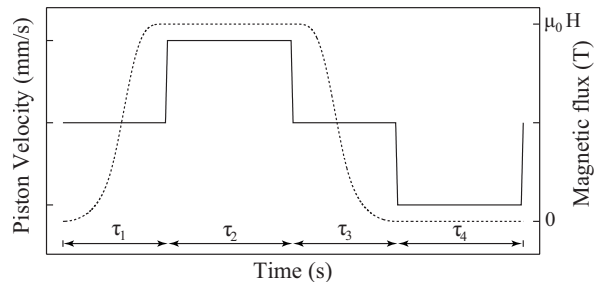

FIG. 2. The magnitude of the magnetic field and velocity of the pistons during the different steps of the refrigeration cycle. The dashed line shows the gradual increase and decrease in the magnetic field and the solid line indicates the movement of the pistons.

in a forthcoming publication. Such a field source would be relevant for a future commercial refrigeration application. However, using an electromagnet allows an easy variation of the applied field making it possible to compare results from the device at different applied fields. Because of the magnetic force from the electromagnet experienced by the regenerator there is a limit to how fast the AMR can be moved in and out of the magnetic field. This, as well as the stray field from the magnet, results in a gradual increase and decrease in the magnetic field and not a stepwise change as in the ideal AMR cycle. Figure 2 shows how the field inside the regenerator increases and decreases during the different steps of the refrigeration cycle, in addition the movement of the pistons is shown.

The times spent for each of the four steps of the AMR cycle are controlled by varying the output of the stepper motors. The time spent on magnetizing, $\tau_{1}$, is set to be identical to the time for demagnetization, $\tau_{3}$. Likewise, the coldto-hot blow, $\tau_{2}$, and the hot-to-cold blow, $\tau_{4}$, are set to take the same time. The total cycle time, $\tau$, is thus twice the sum of $\tau_{1}$ and $\tau_{2}$.

\section{THE NUMERICAL AMR MODEL}

A 2D mathematical model of the magnetic refrigeration device has been developed, which is described in detail in Ref. 8. The numerical model solves the mass and momentum equations for the flow of the heat transfer fluid and the coupled heat transfer equations for the temperatures in the regenerator and in the fluid. The only inputs to the model are the physical properties of the modeled materials. Thus, there are no adjustable parameters. The model is based on the finite element method and implemented using the COMSOL Multiphysics software package. ${ }^{12}$ By neglecting boundary effects in the transversal direction the geometry shown in Fig. 3 can be confined to two dimensions. Neglecting boundary effects in the vertical direction the model geometry may be reduced by symmetry to half a magnetocaloric sheet coupled to half a heat transfer fluid channel. At either end of the magnetocaloric sheet a heat exchanger, separated from the magnetocaloric sheet by a small gap, is included in the model. The heat exchangers are assumed to be in contact with a cooling load and the surroundings. Figure 3(a) shows a schematic of the full 2D geometry considered in the development of the mathematical model and Fig. 3(b) illustrates the simplification of the full model geometry into the repeating unit. 


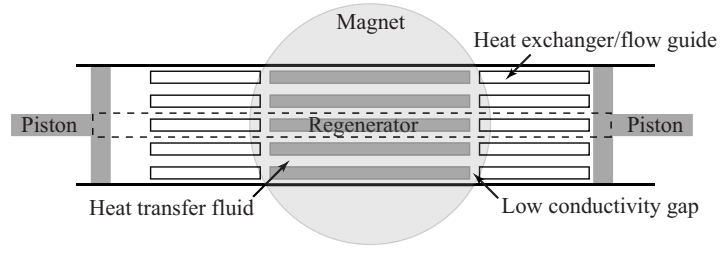

(a)

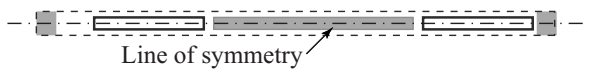

(b)

FIG. 3. (a) The full model geometry of the numerical AMR model. The magnetic field is perpendicular to the plane of the paper, i.e., in the plane of the Gd sheets. (b) The repetitive design of the regenerator allows the full geometry to be reduced to a repeating unit. Because of the symmetry (illustrated by the dotted line) of the repeating unit the final model geometry can be reduced to half a regenerator sheet, fluid channel, and heat exchanger/ flow guide.

The experimental AMR does not include the heat exchangers present in the model geometry. However, the experimental device does implement two flow guides placed at the positions of the heat exchangers in the model which are in contact with the Perspex tubes and thus the surroundings [see Fig. 1(b)]. Although the heat conduction through this assembly is low because of the low thermal conductivity of the materials used in the magnetic refrigeration device, the flow guides are used to simulate heat transfer to the surroundings to make the model configuration similar to the experimental setup. The effective thermal conduction associated with half a flow guide was estimated from steady state heat flux numerical analysis assuming relevant material properties. This gives an effective thermal conductance of $k_{\text {eff }}=2.5 \times 10^{-3} \mathrm{~W} \mathrm{~m}^{-1} \mathrm{~K}^{-1}$.

Gadolinium is implemented in the model as the magnetocaloric material using the mean field $\operatorname{model}^{8}$ (MFM) to estimate the heat capacity and adiabatic temperature change. The MFM has been shown to give results in reasonable agreement with experimentally measured results. ${ }^{13,14} \mathrm{~A}$ density of $7900 \mathrm{~kg} \mathrm{~m}^{-3}$ (Ref. 15) and a thermal conductivity of $10.5 \mathrm{~W} \mathrm{~m}^{-1} \mathrm{~K}^{-1}$ (Ref. 15) was assumed for Gd. The specific heat capacity of the plastic flow guides was estimated to be $1500 \mathrm{~J} \mathrm{~kg}^{-1} \mathrm{~K}^{-1}$ and the density was $1300 \mathrm{~kg} \mathrm{~m}^{-3}$.

An ambient temperature of $298 \mathrm{~K}$ was assumed at the hot end and since the cold end of the experimental AMR was insulated with additional foam, the model assumed perfect insulation at the cold end. To compare the experimental and the numerical results, the model evaluates the temperature difference between the cold and the hot ends of the regen- erator, $\Delta T_{\mathrm{HC}}$, by determining the temperature at the positions in the fluid channel which corresponds to thermocouples 1 and 5 [see Fig. 1(b)].

In the model the magnetic field is applied and removed instantaneously. This differs from the gradual application and removal of the magnetic field in the experimental setup as described above. A continuous time varying magnetic field will be implemented in a future version of the model.

\section{RESULTS}

\section{A. Piston stroke variation}

The magnetic refrigeration device has been run using a range of piston strokes from 0.5 to $10 \mathrm{~mm}$. In each experiment $\tau_{1}$ and $\tau_{2}$ are both kept constant at $3 \mathrm{~s}$ each, giving a total cycle time of $12 \mathrm{~s}$. As the piston stroke length is changed, the piston velocity is changed accordingly to ensure a constant $\tau_{2}$. Each piston stroke length is equivalent to moving a fraction of the heat transfer fluid through the regenerator. Due to the difference between the cross sectional area of the Perspex tubes in which the pistons move and the cross sectional areas of the heat transfer fluid channels, a piston stroke of $1 \mathrm{~mm}$ is equivalent to moving the heat transfer fluid $9.5 \%$ of the length of the $\mathrm{Gd}$ sheets.

The electromagnet is set to an applied field of $\mu_{0} H$ $=1.0 \mathrm{~T}$ at the center of the pole gap. Due to the spatial extent of the AMR, it will experience an average field of $\mu_{0} H=0.97 \mathrm{~T}$ when it is centered in the electromagnet, the "in field" position. In the "out of field" position, $130 \mathrm{~mm}$ from the center, the stray field from the electromagnet gives an average field of $\mu_{0} H=0.16 \mathrm{~T}$ within the AMR. This nonzero out of field magnitude is taken into account in the numerical model in order to predict more correctly the temperature distribution within the regenerator. The nonzero field outside the electromagnet results in an effective decrease in the adiabatic temperature change upon magnetization and demagnetization.

A number of different heat transfer fluids have been used in the AMR. The first heat transfer fluid tested was demineralized water, to which $10 \%$ ethanol is added (WE) in order to reduce the surface tension. Ethylene glycol (ethane-1,2-diol) (EG) and propylene glycol (propane-1,2-diol) (PG) are both industrially relevant heat transfer fluids, however with thermal properties that differ significantly from each other and from WE. Finally a commercially obtained extra virgin olive oil (OO) was used as a heat transfer fluid. The properties of each of the heat transfer fluids are given in Table I. These values are used for the model calculations.

TABLE I. Density, $\rho$, heat capacity, $c_{p}$, thermal conductance, $k$, thermal diffusivity, $\alpha=k /\left(\rho c_{p}\right)$, and viscosity, $\mu$, for the heat transfer fluids used in the present study. All parameters are at $20-25{ }^{\circ} \mathrm{C}$.

\begin{tabular}{lcccccc}
\hline \hline Fluid & $\begin{array}{c}\rho \\
\left(\mathrm{kg} \mathrm{m}^{-3}\right)\end{array}$ & $\begin{array}{c}c_{p} \\
\left(\mathrm{~J} \mathrm{~kg}^{-1} \mathrm{~K}^{-1}\right)\end{array}$ & $\begin{array}{c}k \\
\left(\mathrm{~W} \mathrm{~m}^{-1} \mathrm{~K}^{-1}\right)\end{array}$ & $\begin{array}{c}\alpha \\
\left(\mathrm{m}^{2} \mathrm{~s}^{-1}\right)\end{array}$ & $\begin{array}{c}\mu \\
(\mathrm{mPa} \mathrm{s})\end{array}$ & Ref. \\
\hline WE & 981 & 4330 & 0.52 & $1.23 \times 10^{-7}$ & 1.6 & 18 \\
EG & 1115 & 2406 & 0.25 & $0.94 \times 10^{-7}$ & 17.5 & 19 \\
PG & 1036 & 2508 & 0.20 & $0.77 \times 10^{-7}$ & 40.4 & 15 \\
OO & 915 & 2000 & 0.17 & $0.93 \times 10^{-7}$ & 84 & 20 \\
\hline \hline
\end{tabular}




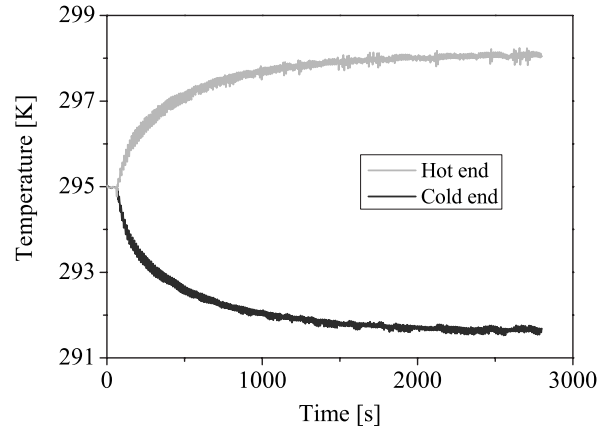

FIG. 4. An example of the raw experimental data from a refrigeration experiment. The temperatures of thermocouples 1 and 5 are plotted as the cold and hot ends, respectively.

In each experiment the temperature span obtained between the cold end and the "hot end" of the AMR is measured when the device has reached steady state. This temperature span is the average difference between thermocouples 1 and 5 and is referred to as $\Delta T_{\mathrm{HC}}$. To compare the experimental and the numerical results, the model evaluates $\Delta T_{\mathrm{HC}}$ by determining the temperatures at the positions in the fluid channel which correspond to thermocouples 1 and 5 . An example of the raw experimental data is shown in Fig. 4. The steady state temperature span for a range of piston stroke lengths using each of the heat transfer fluids are shown in Fig. 5(a). The results obtained from the numerical model for each of the heat transfer fluids in the same range of stroke lengths are shown in Fig. 5(b).

\section{B. Cycle timing variation}

In a second set of experiments, the times spent for the different steps of the AMR cycle have been varied, while keeping the total cycle time and piston stroke length constant. The ratio $\tau_{1} / \tau_{2}$ has been varied in experiments with cycle times of $\tau=12 \mathrm{~s}$ and $\tau=18 \mathrm{~s}$. This is done by introducing a wait time after the magnetization and demagnetization to prolong $\tau_{1}$ and adjusting the piston velocity in order to vary $\tau_{2}$. In all the experiments the piston stroke was such that the heat transfer fluid was moved $50 \%$ of the length of the Gd sheets.

The experiments were run using the water and ethanol mixture as the heat transfer fluid. The temperature span, $\Delta T_{\mathrm{HC}}$, as a function of $\tau_{1} / \tau_{2}$ is shown in Fig. 6. The figure also includes the temperature span predicted by the model for a range of $\tau_{1} / \tau_{2}$ between 0.25 and 4 for cycle periods of both $12 \mathrm{~s}$ and $18 \mathrm{~s}$.

\section{Magnetic field variation}

A set of experiments were performed where the magnitude of the applied field was varied, while keeping the stroke length at $50 \%$ and a cycle time of $12 \mathrm{~s}$ with a ratio of $\tau_{1} / \tau_{2}=1$. The applied field was varied in a range from $\mu_{0} H$ $=0.30 \mathrm{~T}$ to $\mu_{0} H=1.30 \mathrm{~T}$. The steady state temperature difference measured in the AMR and predicted by the model is shown as a function of the magnetic field in Fig. 7.

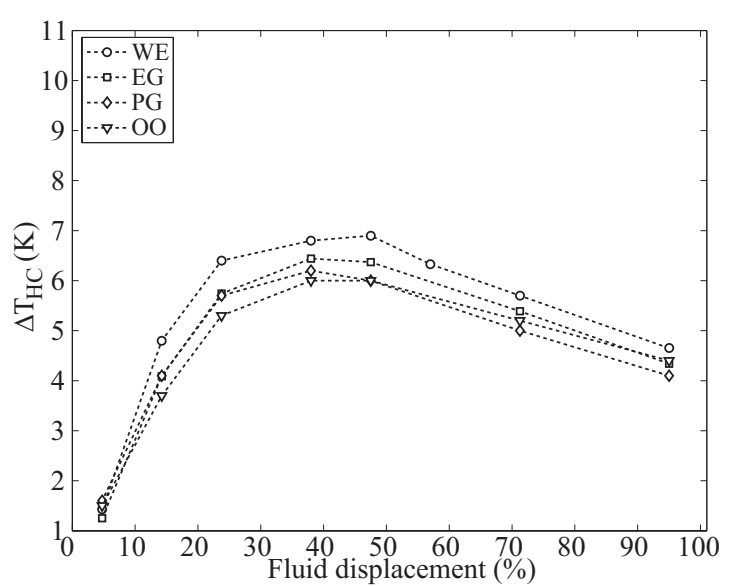

(a)

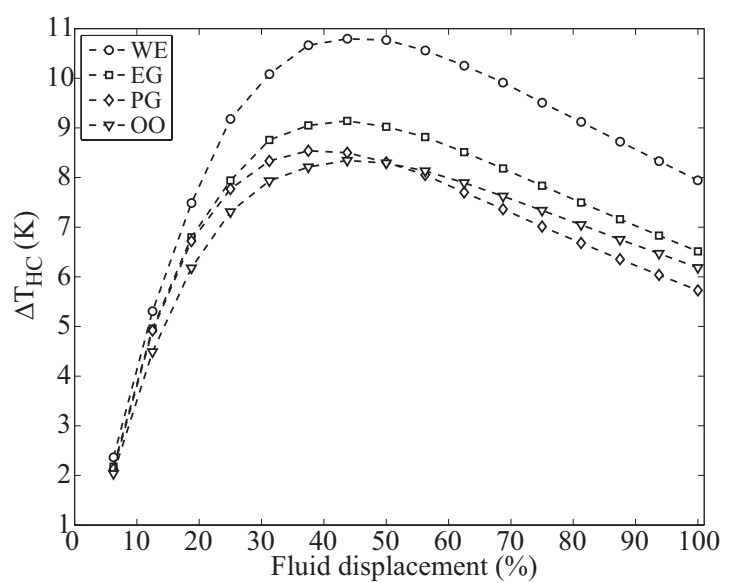

(b)

FIG. 5. The steady state temperature span, $\Delta T_{\mathrm{HC}}$, at a range of piston stroke lengths for a number of different heat transfer fluids. (a) Experimental data obtained from the AMR. (b) Results from the numerical model.

\section{DISCUSSION}

\section{A. Stroke variation}

It is apparent from Figs. 5(a) and 5(b) that the general behavior of $\Delta T_{\mathrm{HC}}$ as a function of stroke length is similar in the experimental data and the model data. Each of the data sets shows how even a small stroke length results in the buildup of a temperature difference between the hot and the cold ends. $\Delta T_{\mathrm{HC}}$ increases sharply as the stroke length in-

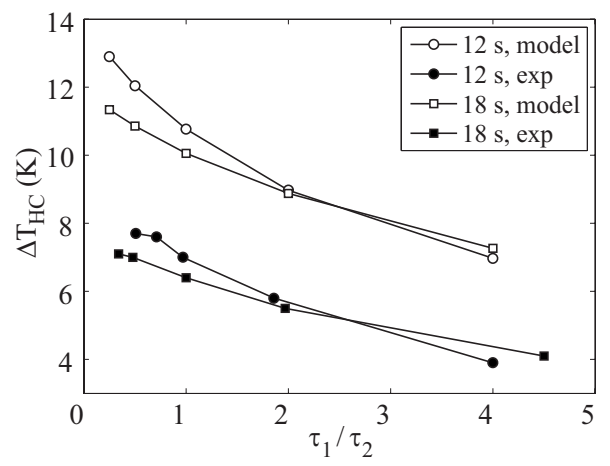

FIG. 6. The steady state temperature span, $\Delta T_{\mathrm{HC}}$, at a range of $\tau_{1} / \tau_{2}$ ratios. The piston stroke length is $50 \%$ and the heat transfer fluid is the water and ethanol mixture. 


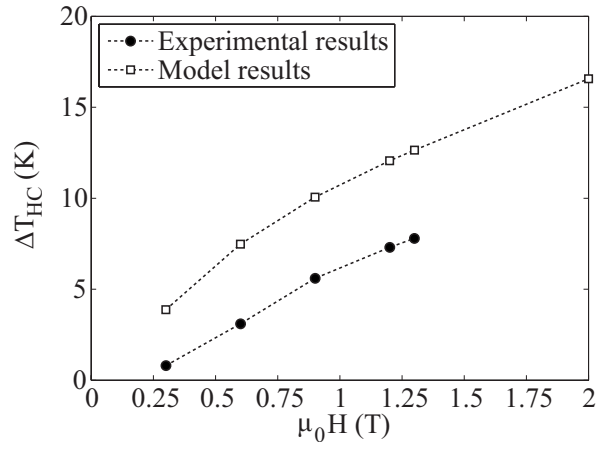

FIG. 7. The steady state temperature span, $\Delta T_{\mathrm{HC}}$, at a range of applied fields. The experimental data are compared to the data obtained from the numerical model.

creases, as more and more fluid participates in the cooling cycle. At a certain fluid displacement the temperature span reaches a maximum. Further increasing the stroke length results in a gradual decrease in $\Delta T_{\mathrm{HC}}$. The reason for this is twofold. First, too much fluid is moved, so that increasing amounts of warm fluid is pushed into the cold end and cool fluid is pushed into the hot end, destroying the regeneration and reducing $\Delta T_{\mathrm{HC}}$. Second, in order to maintain a constant $\tau_{2}$ the fluid is moved faster at long piston stroke lengths resulting in an insufficient heat transfer from the Gd to the heat transfer fluid. The maximum $\Delta T_{\mathrm{HC}}$ obtained for each of the four fluids, along with the corresponding fluid displacement is given in Table II. For each of the fluids a good agreement on the optimum fluid displacement is observed between the experimental data and the model predictions.

In both the experimental results and the model calculations a crossover of $\Delta T_{\mathrm{HC}}$ for $\mathrm{OO}$ and $\mathrm{PG}$ is observed at around $50 \%$ fluid displacement. This may be due to the lower thermal diffusivity of PG compared to OO. As the stroke length increases the velocity of the heat transfer fluid must increase to maintain a constant $\tau_{2}$. The higher thermal diffusivity of $\mathrm{OO}$ facilitates a rapid adjustment of the temperature to that of the $\mathrm{Gd}$, giving a better regeneration and thus a larger value of $\Delta T_{\mathrm{HC}}$.

The model consistently predicts a larger maximum temperature span than observed in the experimental data. An important reason for this may be a reduction in the adiabatic temperature change in the $\mathrm{Gd}$ due to the presence of impurities. The mean field model predicts a $\Delta T_{\mathrm{ad}}$ in fairly good agreement with that measured in highly pure Gd. However, measurements of a commercial grade Gd sample, similar to the one used in the present study, resulted in a reduction in $\Delta T_{\text {ad }}$ of approximately $20 \% .{ }^{16}$ As will be shown below this

TABLE II. The maximum temperature span, $\Delta T_{\mathrm{HC}}$, and corresponding fluid displacements for each of the heat transfer fluids tested.

\begin{tabular}{lcccc}
\hline \hline & \multicolumn{2}{c}{ Experimental } & \multicolumn{2}{c}{ Model } \\
Fluid & $\begin{array}{c}\text { Max. } \Delta T_{\mathrm{HC}} \\
(\mathbf{K})\end{array}$ & $\begin{array}{c}\text { Displacement } \\
(\boldsymbol{\%})\end{array}$ & $\begin{array}{c}\text { Max. } \Delta T_{\mathrm{HC}} \\
(\mathbf{K})\end{array}$ & $\begin{array}{c}\text { Displacement } \\
(\boldsymbol{\%})\end{array}$ \\
\hline WE & 6.9 & 48 & 10.8 & 44 \\
EG & 6.4 & 38 & 9.1 & 44 \\
PG & 6.2 & 38 & 8.5 & 38 \\
OO & 6.0 & 48 & 8.3 & 44 \\
\hline \hline
\end{tabular}

would result in a reduction of approximately $20 \%$ in the predicted values of $\Delta T_{\mathrm{HC}}$.

A further reason for the overestimation of the temperature span is the gradual application and removal of the field in the experimental setup. As the sheets of Gd are moved in and out of the magnet, the field sweeps along the sheets. This creates an unwanted nonuniform temperature gradient within the sheets resulting in an increased diffusion of heat along the length of the sheets. From the thermal conductivity of Gd and the geometry of the AMR the diffusion along the temperature gradient of the sheets will be about $77 \mathrm{~mW} \mathrm{~K}^{-1}$. Any increased temperature gradient in the sheets, due to the gradual application of the magnetic field, will contribute some fraction of this value as a loss. This will reduce the overall gradient in the plates and thus the temperature difference between the hot and the cold end, $\Delta T_{\mathrm{HC}}$. The magnitude of the reduction depends on the duration of the field sweep, i.e., $\tau_{1}$, which in all the present experiments is of the order of the heat diffusion time of the sheets. This reduction is not captured by the model where an instantaneous application and removal of the field is assumed. A future version of the numerical model will take the gradual field sweep along with the reduction in $\Delta T_{\text {ad }}$ due to impurities into account.

Finally, there are heat losses to the surroundings in the experimental setup which are not included in the model. Such heat losses, also leading to an overestimation of the temperature span by the model, may be estimated from the material properties of the device to be of the order of $40 \mathrm{~mW} \mathrm{~K}^{-1}$. Heat losses to the surroundings are augmented by a large thermal conductance of the heat transfer fluid as well as the temperature difference between the heat transfer fluid and the surroundings. The thermal conductivity of WE is more than twice the value of any of the other fluids. Also, the temperature span obtained with WE is larger than with the other fluids. Thus, with WE as the heat transfer fluid the performance of the AMR is expected to be overestimated the most. As seen in Table II this is indeed the observed result.

Previous investigations have shown that the cooling capacity of an AMR decreases linearly with the temperature span. ${ }^{17}$ The present device is operated without a heat load. However, very preliminary results from the recent implementation of such a heat load indicate an inclination of the cooling capacity of the order of $-0.15 \mathrm{~W} \mathrm{~K}^{-1}$, strongly dependent on the experimental conditions. The losses discussed above serve to offset the origin of the cooling capacity. A more advanced model including these losses is under development and will be presented in a future publication.

\section{B. Cycle timing variation}

In the cycle timing variation data, Fig. $6, \Delta T_{\mathrm{HC}}$ is observed to increase as $\tau_{1} / \tau_{2}$ is decreased. This is true for both the experimental data and the model predictions with cycle periods of both 12 and $18 \mathrm{~s}$. Again the model consistently overestimates the temperature span of the AMR. The heat transfer fluid employed was WE with a fluid displacement of $50 \%$, so in accordance with the above results an overestimation is expected due to the large thermal conduction of WE. This is in addition to the overestimation of the adiabatic temperature change due to impurities in the $\mathrm{Gd}$ and the 
gradual removal of the magnetic field discussed above. Both the reduced $\Delta T_{\mathrm{ad}}$ due to impurities and the heat loss will depend on $\Delta T_{\mathrm{HC}}$ and decrease as the $\Delta T_{\mathrm{HC}}$ decreases. Conversely, the relative overestimation of $\Delta T_{\mathrm{HC}}$ due to the gradual movement of the regenerator in and out of the field will increase as $\tau_{1}$ is increased. This is the reason for the almost constant absolute difference between the experimental results and the model predictions.

Figure 6 shows for both data sets that below a certain value of $\tau_{1} / \tau_{2}$ a cycle period of $12 \mathrm{~s}$ gives a higher $\Delta T_{\mathrm{HC}}$ than a cycle period of $18 \mathrm{~s}$. The slower $18 \mathrm{~s}$ cycle has a larger heat loss to the surroundings during the cycle and also more thermal conduction within the AMR. When decreasing $\tau_{1} / \tau_{2}$, there will be an increase in $\tau_{2}$. The resulting slower movement of the liquid during $\tau_{2}$ allows thermal equilibrium between the gadolinium and fluid to be established. At large values of $\tau_{1} / \tau_{2}$ the time $\tau_{2}$ becomes short giving a poor performance, as thermal equilibrium is not reached in each cycle. This is especially true for the $12 \mathrm{~s}$ cycle where $\tau_{2}$ is very short for large values of $\tau_{1} / \tau_{2}$. This very short $\tau_{2}$ explains the crossover observed in both the experimental data and the model predictions, where the $18 \mathrm{~s}$ cycle gives a higher $\Delta T_{\mathrm{HC}}$ than a $12 \mathrm{~s}$ cycle at the largest values of $\tau_{1} / \tau_{2}$.

It is apparent in both the experimental data and the model predictions that $\tau_{1}$ should be as small as possible. Any excess time spent on $\tau_{1}$ leads to a poorer performance due to dissipation of heat to the surroundings. An example is seen by comparing the $\tau_{1} / \tau_{2}=2$ model data point in the $18 \mathrm{~s}$ cycle with the $\tau_{1} / \tau_{2}=1$ model data point for the $12 \mathrm{~s}$ cycle. At both data points $\tau_{2}=3 \mathrm{~s}$ while $\tau_{1}=3 \mathrm{~s}$ in the $12 \mathrm{~s}$ cycle and $\tau_{1}$ $=6 \mathrm{~s}$ in the $18 \mathrm{~s}$ cycle. The shorter $\tau_{1}$ results in a higher $\Delta T_{\mathrm{HC}}$. This would suggest that a large $\tau_{2}$ is beneficial. However, increasing $\tau_{2}$ beyond a certain value does not result in an increase in $\Delta T_{\mathrm{HC}}$. This is seen by comparing the $\tau_{1} / \tau_{2}$ $=0.5$ model data point in the $18 \mathrm{~s}$ cycle with the $\tau_{1} / \tau_{2}=1$ model data point for the $12 \mathrm{~s}$ cycle. At both data points $\tau_{1}=3 \mathrm{~s}$ while $\tau_{2}=3 \mathrm{~s}$ in the $12 \mathrm{~s}$ cycle and $\tau_{2}=6 \mathrm{~s}$ in the 18 $\mathrm{s}$ cycle. The two data points result in almost identical values of $\Delta T_{\mathrm{HC}}$, so there is no advantage in prolonging $\tau_{2}$ from 3 to $6 \mathrm{~s}$.

The model may be used to calculate the temperature profile within the heat transfer fluid in the regenerator. This has been done for the $\tau_{1} / \tau_{2}=0.5$ data in both the $12 \mathrm{~s}$ cycle and $18 \mathrm{~s}$ cycle, as shown in Fig. 8. The temperature profiles are calculated at the steady state temperature span, directly after the cold-to-hot blow. The profiles may be compared to the experimental data at the values of $\tau_{1} / \tau_{2}$ close to 0.5 . The temperatures of each of the five thermocouples are indicated in both plots. As it is expected, both the measured and the calculated temperatures follow an almost linear gradient within the fluid. The calculated gradients are of course steeper due to the larger $\Delta T_{\mathrm{HC}}$ in the model data compared to the measured data.

\section{Field variation}

As expected $\Delta T_{\mathrm{HC}}$ increases in both the experimental data and the model predictions as the applied field is increased. Again it is observed that the model consistently pre-

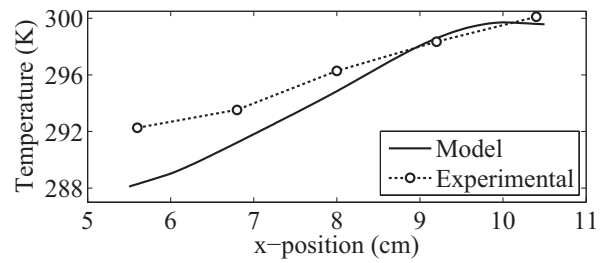

(a)

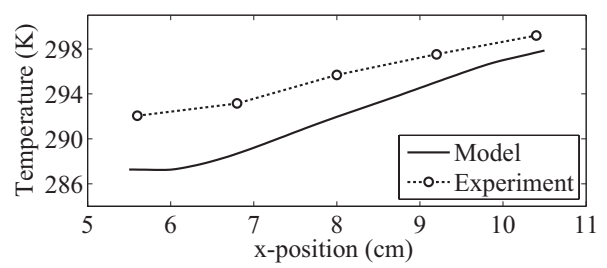

(b)

FIG. 8. The temperature profiles within the heat transfer fluid at steady state for cycle periods of (a) $12 \mathrm{~s}$ and (b) $18 \mathrm{~s}$. Both data sets are at $\tau_{1} / \tau_{2}=0.5$. The solid lines indicate the temperature profiles calculated by the model, while the open data points indicate the experimentally measured data points, connected by a dashed line for clarity.

dicts a higher $\Delta T_{\mathrm{HC}}$ than measured in the experiments. This is due to the same reasons as discussed above.

The geometry of the Gd sheets gives rise to a demagnetizing field opposing the applied field, thus reducing the internal field experienced by the Gd. The magnitude of such a field is minimized by the orientation of the field in the plane of the sheets. However, due to the high susceptibility of Gd in low fields the demagnetization field will still be significant. The magnetic field used as an input to the numerical model is the internal field. Thus the experimental data in Fig. 7 move toward smaller fields compared to the model results, further reducing the difference between the experimental data and model predictions.

The mean adiabatic temperature change in the Gd plates at steady state may be calculated from the temperature dependence of $\Delta T_{\mathrm{ad}}$ assuming the linear temperature gradient in the Gd plates, as observed above, using MFM. An effective adiabatic temperature change, $\Delta T_{\text {eff }}$ is calculated as the average of the mean adiabatic temperature changes in the $\mathrm{Gd}$ sheets upon magnetization and demagnetization.

This effective adiabatic temperature change, along with the steady state temperature span, $\Delta T_{\mathrm{HC}}$, may be used to define a ratio of regeneration, $\zeta$, as

$$
\zeta=\frac{\Delta T_{\mathrm{HC}}}{\Delta T_{\mathrm{eff}}} .
$$

This is a measure of how much the adiabatic temperature change induced by magnetization and demagnetization is amplified by the AMR. Figure 9 shows $\zeta$ as a function of applied field for both the model and experimental data. Interestingly, a constant value of $\zeta$ of just below 4 is observed in the model data independent of the applied field.

In the experimental data, however, the value of $\zeta$ gradually increases until it reaches a constant value of about 2.4 at around $1 \mathrm{~T}$. This constant value is lower than the one observed in the model prediction due to the same reasons as outlined above. 


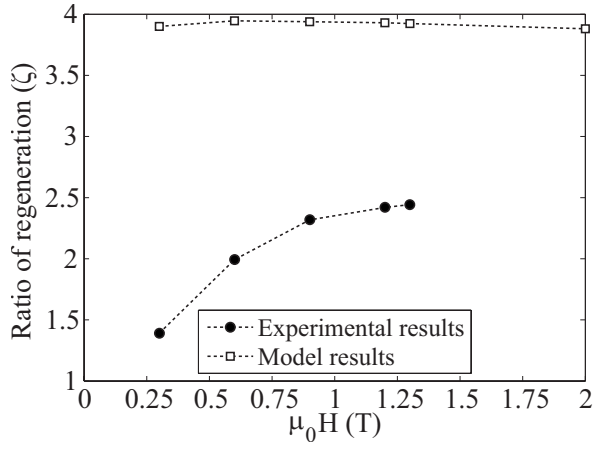

FIG. 9. The ratio of regeneration as a function of the applied field for both the model calculations and the experimental data.

The initially lower value of $\zeta$ may be due to other losses such as mixing, which are not present in the model calculations. Again, the numerical model uses the internal magnetic field, whereas the experimental data are plotted as a function of the applied magnetic field. Thus the experimental data in Fig. 9 move toward smaller fields compared to the model results.

\section{CONCLUSION}

A versatile magnetic refrigeration test device allowing control of a large number of experimental parameters has been presented. Results from parameter variations of the type of heat transfer fluid, the heat transfer fluid movement, cycle timing, and applied magnetic field are given. These results are compared to the results obtained from a detailed 2D numerical model of the magnetic refrigeration test device. A qualitative and, to some extent, quantitative agreement between experiment and model is observed. The deviations between the experiment and model results may be explained by impurities in the gadolinium resulting in a lower adiabatic temperature than implemented in the numerical model, heat losses not included in the model as well as the fact that the gradual movement of the AMR in and out of the field in the experiments is assumed instantaneous in the model.

\section{ACKNOWLEDGMENTS}

The authors would like to thank F. Saxild and K. N. Sørensen for their technical help. Also, the authors would like to acknowledge the support of the Programme Commission on Energy and Environment (EnMi) (Contract No. 2104-06-0032) which is part of the Danish Council for Strategic Research.

${ }^{1}$ Magnetic Refrigeration at Room Temperature, edited by H. Auracher and P. Egolf [special issue of Int. J. Refrig. 29, 1235 (2006)].

${ }^{2}$ K. L. Engelbrecht, G. F. Nellis, S. A. Klein, and C. B. Zimm, HVAC\&R Res. 13, 525 (2007).

${ }^{3}$ K. A. Gschneidner, Jr., V. K. Pecharsky, and A. O. Tsokol, Rep. Prog. Phys. 68, 1479 (2005).

${ }^{4}$ K. A. Gschneidner, Jr., and V. K. Pecharsky, Annu. Rev. Mater. Sci. 30, 387 (2000).

${ }^{5}$ B. F. Yu, Q. gao, B. Zhang, X. Z. Meng, and Z. Chen, Int. J. Refrig. 26, $622(2003)$.

${ }^{6}$ S. J. Lee, J. M. Kenkel, and D. C. Jiles, IEEE Trans. Magn. 38, 2991 (2002).

${ }^{7}$ R. Bjørk, C. H. R. Bahl, A. Smith, and N. Pryds, "Optimization and improvement of Halbach cylinder design,” J. Appl. Phys. 104, 013910 (2008).

${ }^{8}$ T. F. Petersen, N. Pryds, A. Smith, J. Hattel, H. Schmidt, and H.-J. H. Knudsen, Int. J. Refrig. 31, 432 (2008).

${ }^{9}$ T. F. Petersen, K. Engelbrecht, C. R. H. Bahl, B. Elmegaard, N. Pryds, and A. Smith, J. Phys. D 41, 105002 (2008).

${ }^{10}$ V. K. Pecharsky and K. A. Gschneidner, Jr., J. Magn. Magn. Mater. 200, 44 (1999).

${ }^{11}$ J. A. Barclay, J. Alloys Compd. 207, 355 (1994).

${ }^{12}$ See http://www.comsol.com for details on the software package.

${ }^{13}$ S. M. Benford and G. V. Brown, J. Appl. Phys. 52, 2110 (1981)

${ }^{14}$ A. M. Tishin, Cryogenics 30, 127 (1990).

${ }^{15}$ CRC Handbook of Chemistry and Physics, 79th ed., edited by D. Lide (CRC, Boca Raton, FL, 1998).

${ }^{16}$ S. Y. Dan'kov, A. M. Tishin, V. K. Pecharsky, and K. A. Gschneidner, Jr., Phys. Rev. B 57, 3478 (1998).

${ }^{17}$ T. Okamura, K. Yamada, N. Hirano, and S. Nagaya, Int. J. Refrig. 29, 1327 (2006)

${ }^{18}$ Å. Melinder, Thermophysical Properties of Liquid Secondary Refrigerants (International Institute of Refrigeration, Paris, France, 1997).

${ }^{19}$ F. P. Incropera and D. P. DeWitt, Introduction to Heat Transfer (Wiley, New York, 1996).

${ }^{20} \mathrm{See}$ http://www.oliveoilsource.com for physical parameters. 